\title{
A Novel Combination for Graft-versus-Host-Disease Prophylaxis: Lessons Learned from the Birth of Sushi Burrito!
}

\author{
Shahrukh K. Hashmi \\ Department of Internal Medicine, Mayo Clinic, Rochester, MN, USA
}

Innovation has no boundaries, and to achieve a goal in research, one must discover, create, or invent something, or use various combinations of the existing armamentarium (if present) for innovation/discovery. The latter approach is the tale of a novel prophylaxis regimen demonstrated in this issue of Acta Haematologica, where Morozova et al. [1] present data on a pilot trial using the commonly used drugs cyclophosphamide and ruxolitinib for the prevention of graft-versus-host-disease (GVHD) to which this commentary refers to. I use the metaphor of a novel cuisine to demonstrate the novelty of this GVHD regimen.

The tale of innovation in the food industry is interesting, though the pace of innovation and discovery is much slower compared to medical sciences. Occasionally, however, we do see newer recipes on the menu if we were to actively explore the restaurant industry. While newer dishes in the world of gastronomy are rare, we have seen the birth of many hybrid foods in the current millennia, and one such example is the sushi burrito. Sushi, which means "sour-tasting," has been a source of food in Japan for hundreds of years and could be a delicacy in some countries. The origins of burrito, which in Spanish means "little donkey," is attributed to Mexican people thousands

karger@karger.com

(c) 2020 S. Karger AG, Basel

www.karger.com/aha

Karger! of years ago. A combination of these Japanese and Mexican cuisines was a product of this century and is known as "Sushirrito" or simply "sushi burrito." Although no novel recipes are used, the cuisine is novel, and this hybrid food item has gained tremendous popularity all over the world.

Fortunately, such hybrid innovations utilizing the existing materials also occur in healthcare. In the latter half of the twentieth century, when many investigators were trying to develop novel agents in the war against cancer, Emil Frei, James Holland, and Emil Freireich cooked up a recipe of the existing ingredients consistent of steroids, methotrexate, vincristine, and 6-mercaptopurine ("POMP") for the treatment of acute lymphoblastic leukemia, and it was a success [2]. Similarly, for lymphomas, George Canellos and Vincent DeVita prepared a new cuisine with old spices and named it MOPP (nitrogen mustard, vincristine, procarbazine, and prednisone), which was proven to be an effective therapy [3].

Since the inception of hematopoietic cell transplantation (HCT) 7 decades ago by E. Donnall Thomas, the Holy Grail has been the reduction of relapse risk and concurrently avoidance of GVHD. Currently, thousands of trials are underway for the treatment or prevention of 
GVHD globally, but we have not found a panacea. The majority of the trials are of a phase I design and stem from extensive preclinical data (mainly murine). The Hopkins group has championed the approach of alleviating the risk of both acute and chronic GVHD by using posttransplant cyclophosphamide (PTCy) [4], the use of which has skyrocketed over the past decade given its effectiveness in matched related, matched unrelated, and haploidentical transplantations [5-7]. To exploit this approach, Morozova et al. [1] developed a novel regimen for GVHD prophylaxis by utilizing only PTCy and ruxolitinib (45-mg dose given from day 7 to day 2 , and $15 \mathrm{mg}$ from day +5 to day +100 of HCT) after a reduced-intensity conditioned transplant in myelofibrosis patients. They report that out of 20 patients on the trial, 11 patients had severe poor graft function, 1 engraftment failure, and 1 relapse. Grade 3-4 nonhematologic toxicity was observed in $30 \%$, viral reactivation in $45 \%$, and severe sepsis in $15 \%$ of the patients. They observed acute GVHD grade II-IV in 25\%, grade III-IV GVHD in $15 \%$, and moderate chronic GVHD in $20 \%$ of the patients.

A report from the European Society for Blood and Marrow Transplantation of 1,055 patients with myelofibrosis indicated a disease-free survival of $64 \%(60-68)$ and an overall survival of $74 \%(71-78)$ at 10 years (for those who survived the first 2 years post-HCT). Grade IIIV acute GVHD occurred in $23 \%$ of the landmark cohort patients $(n=245)$ in this study, and $56 \%(n=576)$ of the patients (landmark cohort) had chronic GVHD of whom 263 patients had an extensive chronic GVHD [8]. In a phase II multicenter trial of 103 patients with myelofibrosis conditioned with busulfan and fludarabine, the incidence of acute GVHD grade II-IV was $27 \%$, and for chronic GVHD it was $43 \%$ [9]. The results of the study by Morozova et al. [1] compare favorably to the results from the larger studies concerning the incidence of chronic GVHD, without a significant difference in the risk of relapse. However, before applying the novel GVHD prophylaxis regimen in practice, certain issues need to be dealt with first.

The reported study does not provide a clear description of the study phase. A pilot study can be phase I, phase II, phase Ib/IIa study or a small study to test the essential aspects of a clinical trial; e.g., retention strategies, data collection methods, or adherence issues. Generally, a safety study (e.g., classic $3+3+3$ design) evaluates safety parameters as the primary aim, whereas this study had acute GVHD grade II-IV and chronic moderate/severe GVHD as the primary endpoint (clinical outcome). The stopping rules included 4 consecutive or 8 overall cases of grade III-IV acute GVHD and 3 consecutive or 6 overall cases of graft failure. Thus, the overall design was centered on a clinical variable that happens to be the main factor to be targeted by the novel GVHD prophylaxis regimen. Thus, the statistical inference must be drawn with this caveat of an atypical design in mind. About half of the patients in this study suffered from severe poor graft function, which hinders the success of this regimen unless modifications were to occur. Lastly, as in all single-arm studies with a small sample size, the results cannot be applied to clinical practice until randomized trial data are available, since one cannot remove the biases of inferior results from the studies using calcineurin inhibitors as GVHD prophylaxis due to differences in populations.

Zhao et al. [10] recently reported data on the use of ruxolitinib for GVHD prophylaxis in hematologic malignancies resulting in a 1-year cumulative incidence of moderate/severe cGVHD of $21.4 \%$; however, this study was different given no use of PTCy. Kröger et al. [11] reported a reduced risk of acute GVHD in myelofibrosis patients who received peri-transplant ruxolitinib, which, unlike the study by Morozova et al. [1], allowed use of ruxolitinib only until the engraftment post-HCT. Despite the differences in the schema and/or combinations used in these studies, it seems that there is a positive signal that the utilization of ruxolitinib can affect the pathogenesis of GVHD. When combined with PTCy, the effect may even be more pronounced; however, concrete data is still awaited. Despite the imperfect design of the trial, the Morozova et al. study [1] has set the frame for the next phase of clinical trials combining ruxolitinib and PTCy for GVHD prophylaxis and modifying the regimen dose/duration to minimize the risk of poor graft function and graft failure. They should be congratulated to initiate a study that combines already existing agents, with which our transplant community has extensive experience and are probably significantly cheaper compared to the new drugs on the market. Mixing well-known ingredients to create such hybrid regimens (the sushi burrito) may very well lead to scientific breakthroughs in a short period, which can directly affect the clinical practice worldwide, but only time will tell!

\section{Conflict of Interest Statement}

S.K.H. has received honoraria from Novartis, Pfizer, Mallinckrodt, and Janssen. S.K.H. has received travel grants from Sanofi, Gilead, MSD, and GSK. 


\section{References}

1 Morozova EV, Barabanshikova MV, Moiseev IS, Shakirova AI, Barhatov IM, Ushal IE, et al. A Prospective Pilot Study of Graft-versusHost Disease Prophylaxis with Post-Transplantation Cyclophosphamide and Ruxolitinib in Patients with Myelofibrosis. Acta Haematol. DOI: 10.1159/000506758.

2 Rodriguez V, Hart JS, Freireich EJ, Bodey GP, McCredie KB, Whitecar JP Jr, et al. Pomp combination chemotherapy of adult acute leukemia. Cancer. 1973 Jul;32(1):69-75.

3 Schein PS, Chabner BA, Canellos GP, Young RC, Berard C, DeVita VT. Potential for prolonged disease-free survival following combination chemotherapy of non-Hodgkin's lymphoma. Blood. 1974 Feb;43(2):181-9.

4 Luznik L, Fuchs EJ. High-dose, post-transplantation cyclophosphamide to promote graft-host tolerance after allogeneic hematopoietic stem cell transplantation. Immunol Res. 2010 Jul;47(1-3):65-77.

5 Hamadani M, Ahn KW, Litovich C, Herve F, Smith SM, Fenske TS, Boumendil A, et al. Post-transplant cyclophosphamide (PT-Cy) based haploidentical transplantation (haplo-
HCT) versus matched sibling (MSD) or matched unrelated donor (MUD) reduced intensity conditioning (RIC) HCT for diffuse large b-cell lymphoma (DLBCL): A CIBMTR and EBMT analysis. J Clin Oncol. 2018;36(15 suppl):7056-7056.

6 Apperley J, Niederwieser D, Huang XJ, Nagler A, Fuchs E, Szer J, et al. Haploidentical hematopoietic stem cell transplantation: a global overview comparing Asia, the European Union, and the United States. Biol Blood Marrow Transplant. 2016 Jan;22(1):23-6.

7 Robinson TM, Fuchs EJ, Zhang MJ, Labopin M, St Martin A, Keesler DA, et al. Selecting between HLA-Matched Siblings and HLAHaploidentical Related Donors for Acute Leukemia in the Era of Post-Transplant Cyclophosphamide: The Center for International Blood and Marrow Transplant Registry and the Acute Leukemia Working Party of the European Society for Blood and Marrow Transplant. Blood. 2017;130(Suppl 1):851.

8 Robin M, De Wreede LC, Wolschke C, Schetelig J, Eikema DJ, Van Lint MT, et al. 2019. Long-term outcome after allogeneic hematopoietic cell transplantation for myelofibrosis. Haematologica. 2019 Sep;104(9): 1782-88.

9 Kröger N, Holler E, Kobbe G, Bornhäuser M, Schwerdtfeger R, Baurmann H, et al. Allogeneic stem cell transplantation after reducedintensity conditioning in patients with myelofibrosis: a prospective, multicenter study of the Chronic Leukemia Working Party of the European Group for Blood and Marrow Transplantation. Blood. 2009 Dec;114(26): 5264-70.

10 Zhao Y, Shi J, Luo Y, Gao F, Tan Y, Lai X, et al. Calcineurin Inhibitors Replacement by Ruxolitinib as Graft-versus-Host Disease Prophylaxis for Patients after Allogeneic Stem Cell Transplantation. Biol Blood Marrow Transplant. 2020 May;26(5):e128-33.

11 Kröger N, Shahnaz Syed Abd Kadir S, Zabelina T, Badbaran A, Christopeit M, Ayuk F, et al. Peritransplantation ruxolitinib prevents acute graft-versus-host disease in patients with myelofibrosis undergoing allogenic stem cell transplantation. Biol Blood Marrow Transplant. 2018 Oct;24(10):2152-6. 